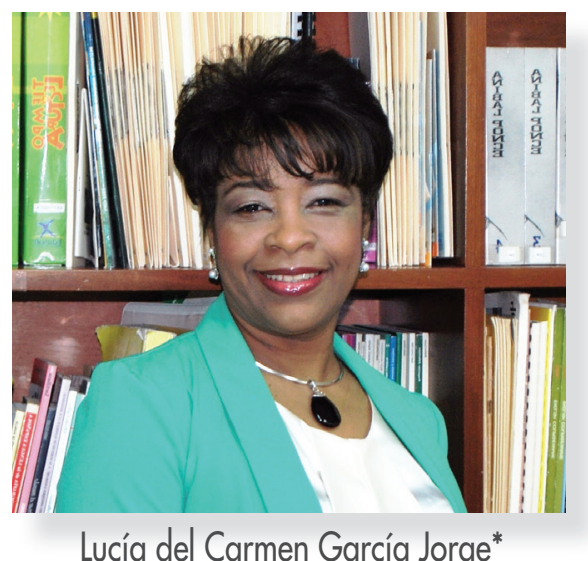

\section{El rol de los tutores en el proceso de escritura de los informes finales de grado}

\section{The role of tutors in writing undergraduate theses}

Recibido: 07-11-17

Aprobado: 02-01-18

\title{
Resumen
}

Este trabajo se enmarca en un programa piloto llevado a cabo en la Pontificia Universidad Católica Madre y Maestra (PUCMM) para apoyar el desarrollo escrito de los trabajos finales de grado, realizado en el transcurso de un año (mayo 2016-mayo 2017) con docentes y estudiantes de las carreras de Comunicación Social, Arquitectura y Gestión Financiera y Auditoría. El propósito fundamental es presentar el impacto de la labor de los tutores en el trabajo con tesistas, a partir del proceso formativo realizado y de su función de servir de apoyo a los asesores disciplinares, orientando el proceso de escritura de informes finales de grado de los estudiantes que concluyen sus carreras. Se basa en los conceptos de alfabetización académica (Carlino, 2011), de escritura como proceso de construcción de significados (Cassany, 2003) y como práctica ejercida en situaciones específicas (Moyano y Natale, 2012). Asimismo, en la consideración del rol de los comentarios escritos en la construcción de la tesis (Tapia-Ladino, Arancibia, y Correa, 2016). Este estudio presenta el trabajo realizado para la selección de los estudiantes tutores, el modelo de formación (con sesiones presenciales, acompañamiento en el aula, seguimiento por parte de un miembro del Equipo Técnico del Centro de Excelencia para la Investigación y Difusión de la Lectura y la Escritura -CEDILE) y las acciones pedagógicas implementadas con los profesores asesores para la acción conjunta. Se exponen y analizan, además, los resultados alcanzados hasta el momento, destacando las fortalezas y compartiendo los problemas que se enfrentaron durante la puesta en práctica del programa piloto. Como resultado de esta experiencia, fueron impactados 86 estudiantes tesistas, quienes mejoraron la calidad de sus informes profesionales de grado.

\section{Abstract}

This research article describes the initial findings of a pilot program that provided eighty six Communication, Architecture, and Financial Management \& Auditing students at the Pontificia Universidad Católica Madre y Maestra with guidance on how to write their undergraduate theses. The program, grounded in the concepts of academic literacy (Carlino, 2011) and writing as a process of construction of meanings (Cassany, 2003) and as practice exercised in specific situations (Moyano and Natale, 2012), began in May of 2016 and lasted a full year. This study presents the method used for the selection of student tutors, the training model (which included face-to-face sessions, classroom group work, and follow-ups by a member of the Technical Team of the Center of Excellence for Research and Reading Dissemination - CEDILE), and the pedagogical actions implemented together with the students' formal thesis advisors. It presents an analysis of the program results, including a discussion on both the successes and setbacks encountered in the implementation process.

\section{Palabras clave}

Trabajos finales de grado; proceso de escritura; formación de tutores; modelo de capacitación; acompañamiento

\section{Keyword}

undergraduate theses; writing process; training of tutors; training model; mentoring

\footnotetext{
*Lucía del Carmen García Jorge: Magíster en Lingüística Aplicada por la Pontificia Universidad Católica Madre Y Maestra. También es profesora del Departamento de Español en la misma universidad. Ha coordinado el Programa Nacional de Lectura, Escritura y Matemática MINERD-PUCMM (2007-2009) y se ha se desempeñado como coordinadora de capacitación del Programa Escuelas Efectivas Lengua Española (PEF-2009-2014). Para contactar a la autora: Igarcia@pucmm.edu.do
} 


\section{Introducción}

La producción de los informes finales de grado representa un esfuerzo cognitivo para los estudiantes que se encuentran al término de su carrera, debido a que no cuentan con las herramientas necesarias para la construcción de este género académico, pues no han trabajado la escritura a lo largo de su formación disciplinar. Las investigaciones han demostrado que escribir es un proceso complejo, incluso para los escritores expertos, pues esta actividad requiere el uso de estrategias cognitivas y metacognitivas, las cuales deben ser aprendidas y, por tanto, también enseñadas. No obstante, aunque los profesores asesores representan el saber disciplinar, muchas veces no cuentan con las herramientas lingüísticas necesarias para modelar este tipo de estrategias. Respecto a la escritura, Carlino (2011) afirma:

Escribir es una herramienta para pensar y no solo es un canal de comunicación. Es un instrumento que sirve a la humanidad para resolver problemas: permite registrar información y darle permanencia en el tiempo, extiende la memoria humana, amplía la capacidad del cerebro... la escritura en cuanto medio de comunicación posibilita contactarse con otros que no están físicamente presentes, la función más conocida de la escritura es la función comunicativa: se escribe para comunicar a otros algo que inicialmente es de uno. El que escribe empieza a promocionarse profesionalmente. (pp. 95-96)

Significa, entonces, que la docencia universitaria tiene un gran reto por delante, pues le corresponde desarrollar en sus estudiantes las competencias necesarias que les permitan producir textos académicos para desempeñarse en su saber disciplinar.

Esto implica orientar el proceso de escritura tomando en cuenta un propósito, un destinatario y un contexto; saber para qué va escribir, a quién le va escribir, por qué va escribir. Sin embargo, los estudiantes tesistas no siempre tienen las respuestas a estas interrogantes. Asimismo, con relación a esta problemática, Giudice, Godoy y Moyano, (2016) afirman que:

...los textos que los estudiantes tienen que abordar en su paso por la universidad son propios de una comunidad discursiva (Swales,1990) que, como tal, se caracterizan por disponer de formas particulares de comunicación. Estos géneros resultan extraños y ajenos a la mayoría de los estudiantes, en tanto definen prácticas que se circunscriben a un ámbito desconocido para ellos, en el que sus conocimientos y experiencias escolares no son suficientes. Nuevos ámbitos implican la necesidad de aprender nuevos géneros. (p. 3)
En ese sentido, los estudiantes universitarios de la PUCMM, al final de su carrera tienen que realizar un trabajo de investigación que los acreditará como profesionales en un área disciplinar, lo que implica utilizar una serie de estrategias para la producción de un género del discurso académico; en este caso, la tesis de grado. Es en este contexto en el que surge la propuesta del CEDILE-PUCMM referida a un programa de formación, tanto para los profesores asesores de las carreras de Comunicación Social, Arquitectura y Gestión Financiera y Auditoría, como para estudiantes tutores, a través de encuentros talleres donde se desarrollan diferentes prácticas para fortalecer las habilidades en los procesos de planificación, textualización, revisión y edición de la escritura de los informes finales de grado. El propósito esencial de este programa es que los tutores brinden apoyo a los estudiantes tesistas en el proceso de escritura de los trabajos finales de grado. Para lograrlo, los tutores reciben una formación que incluye horas de capacitación presenciales y virtuales, acompañamiento y seguimiento al trabajo realizado. "El proceso de escribir se comprende mejor como un conjunto de diferentes procesos de pensamiento que el escritor regula y organiza durante este acto". (Cassany, 2003)

A continuación, se presenta el proceso llevado a cabo para la selección de los tutores, el perfil requerido, los aspectos tomados en cuenta para su formación y el acompañamiento realizado por un miembro del equipo técnico del CEDILE para afinar y apoyar el proceso. También se exponen los indicadores utilizados para el apoyo a los estudiantes tesistas y la valoración que muestra el grado de satisfacción sobre la ayuda recibida del tutor y el profesor asesor.

\section{Desarrollo}

\section{Selección de los tutores}

La selección de un tutor para la tarea de acompañar el proceso de escritura a estudiantes que escriben sus informes finales de grado requiere tener en cuenta ciertas competencias específicas. Entre ellas, poseer habilidades tanto de comprensión como de producción escrita, así como buen desarrollo de las relaciones interpersonales, pues este trabajo entre pares debe realizarse en un ambiente afectivo colaborativo. De acuerdo a Molina: ...las tutorías entre pares establecen una
forma de interacción situada en un contexto
específico, en el cual los roles de tutor y tutorado
los sitúan como sujetos discursivos que traen
realidades y experiencias que comparten para
construir una tarea común. En esta interacción,
las subjetividades de ambos establecen
negociaciones para definir el objetivo que se va
a construir de manera colaborativa. (2016, p.18) 
Para la conformación de un grupo de tutores de la PUCMM, se incentivó la participación de estudiantes de la comunidad universitaria a través de la página web del CEDILE y atendiendo a los siguientes requerimientos:

Tabla 1: Requerimientos para concursar como tutor

\begin{tabular}{|l|}
\hline \multicolumn{1}{|c|}{ Requerimientos para concursar como tutor } \\
\hline Vocación de servicio y facilidad para comunicarse e interactuar con los demás. \\
\hline $\begin{array}{l}\text { Desarrollo pleno de las habilidades de lectura y escritura antes de su llegada a la PUCMM, } \\
\text { certificada por sus notas del Bachillerato. }\end{array}$ \\
\hline Placer por la lectura y la escritura. \\
\hline Dominio amplio de la normativa: acentuación, puntuación, uso de letras, concordancia. \\
\hline $\begin{array}{l}\text { Haber cursado como mínimo los tres primeros semestres de la PUCMM y contar con un buen } \\
\text { índice académico en la Universidad. }\end{array}$ \\
\hline Buenas referencias de sus profesores universitarios anteriores. \\
\hline Disponibilidad de tiempo para apoyar a otros estudiantes en sus escritos. \\
\hline Disposición para la formación continua. \\
\hline
\end{tabular}

Fuente: CEDILE

Ante la primera convocatoria, se presentaron 15 candidatos, de los cuales solo 14 concursaron. Estos candidatos están inscritos en las siguientes carreras: dos estudiantes de Educación; dos de Comunicación Social; dos de Arquitectura; dos de Ciencias Jurídicas y uno de Administración de Empresas. A estos participantes se les aplicó una prueba de comprensión, con diferentes tipos de preguntas que evaluaban tanto el nivel inferencial como el nivel crítico. También se les suministró una prueba de producción escrita para elaborar un ensayo expositivo, a partir de una consigna detallada y con criterios de evaluación específicos, que se entregó previamente a su escritura. Luego, se les realizó una entrevista para determinar con mayor claridad su perfil. De este grupo, 9 estudiantes calificaron, pero solo 7 fueron contratados, pues dos de ellos no pudieron participar de esta experiencia por asuntos de tiempo. En una nueva convocatoria, se realizó el mismo proceso, constituyéndose un segundo grupo de 10 estudiantes tutores, con lo que el grupo final se elevó a 17.

\section{Perfil del tutor acompañante}

Molina (2016, p. 18) define las tutorías entre pares como "una estrategia de aprendizaje colaborativo que se emplea en los centros de aprendizajes o de escritura de muchas universidades". De acuerdo a la autora, estas tutorías se caracterizan por un trabajo individualizado y dirigido a atender las necesidades de escritura planteadas por el tutorado. Visto de esta manera, se requiere entonces, que el tutor posea un perfil de acompañante que le permita orientar el proceso de escritura a los estudiantes tesistas de las diferentes carreras de grado seleccionadas: Comunicación Social, Arquitectura y Gestión Financiera y Auditoría.
A continuación, destacamos el perfil deseado para cada tutor del programa.

- Dominio de las competencias básicas de escritura.

- Conocimientos sobre el proceso cognitivo de la escritura y las estrategias para implementarlo en la práctica.

- Conocimiento, capacidad y actitud positiva para que su rol sea aceptado y legitimado con credibilidad.

- Disposición para recibir formación continua para mejorar su rol en el proceso de acompañamiento a estudiantes tesistas.

- Compromiso con el mejoramiento del proceso de producción escrita de los trabajos finales de grado.

- Atención a las necesidades particulares del tesista, con sugerencias oportunas para que cada estudiante revise y edite su propio trabajo.

- Apoyo al desarrollo de estrategias cognitivas y metacognitivas por parte de los tesistas.

- Relación empática y positiva, basada en la confianza, y no afectada por el poder o la consideración de superioridad de parte del acompañante.

- $\quad$ Actitud de objetividad y equilibrio.

- Respeto a la propiedad intelectual y a los derechos de autor.

- Responsabilidad, puntualidad y respeto al principio de confidencialidad.

Para lograr este perfil, se estableció un proceso formativo que completó el grupo de tutores seleccionados y que describimos a continuación. 


\section{Proceso de formación a los estudiantes tutores}

Tabla 2: Programa de formación de los tutores

\begin{tabular}{|c|c|}
\hline & Programa formativo \\
\hline \multirow[t]{4}{*}{ Primera etapa } & $\begin{array}{c}\text { Taller 1: - Concepciones de lectura y escritura. } \\
\text { - Valor epistémico de la escritura. }\end{array}$ \\
\hline & Taller 2: - Proceso de escritura en los trabajos finales de grado. \\
\hline & $\begin{array}{l}\text { Taller 3: - El género discursivo. } \\
\text { - Situación de enunciación y estructura del género discursivo: } \\
\text { informe final de grado. }\end{array}$ \\
\hline & $\begin{array}{l}\text { Taller 4: - El informe profesional (Comunicación Social y Gestión } \\
\text { Financiera y Auditoría) y el Informe de proyecto de grado } \\
\text { (Arquitectura) como géneros académicos. } \\
\text { - Estructura del informe profesional y del informe de proyecto } \\
\text { de grado, consensuada con los docentes de cada } \\
\text { Departamento involucrado. }\end{array}$ \\
\hline \multirow[t]{4}{*}{ Segunda etapa } & $\begin{array}{l}\text { Taller 5: - Evaluación de la escritura de los informes finales de grado. } \\
\text { - Instrumentos de evaluación de la escritura para los informes } \\
\text { finales de grado, consensuados con los docentes de cada } \\
\text { Departamento involucrado. }\end{array}$ \\
\hline & $\begin{aligned} \text { Taller 6: - Perfil del tutor acompañante. } \\
\text { - Criterios e indicadores para evaluar el proceso de } \\
\text { seguimiento a los estudiantes tesistas. }\end{aligned}$ \\
\hline & Taller 7: - Procedimientos de coherencia. \\
\hline & Taller 8: - Procedimientos de cohesión. \\
\hline \multirow[t]{2}{*}{ Tercera etapa } & Taller 9: - El proceso de revisión de los trabajos finales de grado. \\
\hline & $\begin{array}{l}\text { Taller 10: - Cómo citar en el informe final de grado según formato } \\
\text { APA. } \\
\text { - La polifonía en los trabajos finales de grado. }\end{array}$ \\
\hline
\end{tabular}

Fuente: CEDILE

Acompañamiento durante el proceso de escritura

En el programa desarrollado, el tutor es un "acompañante" que colabora con estudiantes-tesistas durante el proceso de redacción de los trabajos finales de grado en sus fases de planificación, textualización revisión y edición, guiado por el profesor asesor de la carrera correspondiente y apoyado por el CEDILE. Se espera que el tutor-acompañante, más que detectar problemas y ofrecer soluciones, participe en la identificación de fortalezas y aspectos a mejorar en los trabajos escritos que asiste. Además, que realice intervenciones pertinentes para responder a las necesidades puntuales de cada tesista en particular, a fin de que este mejore sus competencias al momento de producir su texto escrito y reflexione sobre su propio proceso de escritura.
En este sentido, acompañar significa estar cerca, apoyar, gestionar, cualificar las fortalezas y canalizar las necesidades y aspectos a mejorar del acompañado, siempre de la mano del profesor asesor y con el apoyo del CEDILE. Durante este proceso, se espera que el tutor sea eficaz en el uso del tiempo acordado para cada proyecto al que da seguimiento (una hora de tutoría en cada encuentro, con un total de veinte horas durante un período académico).

Para el desarrollo de esta etapa, se elaboró un instrumento que evalúa las fases del proceso de escritura llevado a cabo por los tesistas y orientado por los tutores. Con el mismo se dio seguimiento a los trabajos elaborados para fortalecer el proceso de "textualización" con realimentación dirigida a mejorar la calidad del producto final: la tesis de grado. A continuación, presentamos el instrumento utilizado con sus indicadores: 
Instrumento utilizado para el acompañamiento al trabajo de los tesistas

Tabla 3: Instrumento de acompañamiento

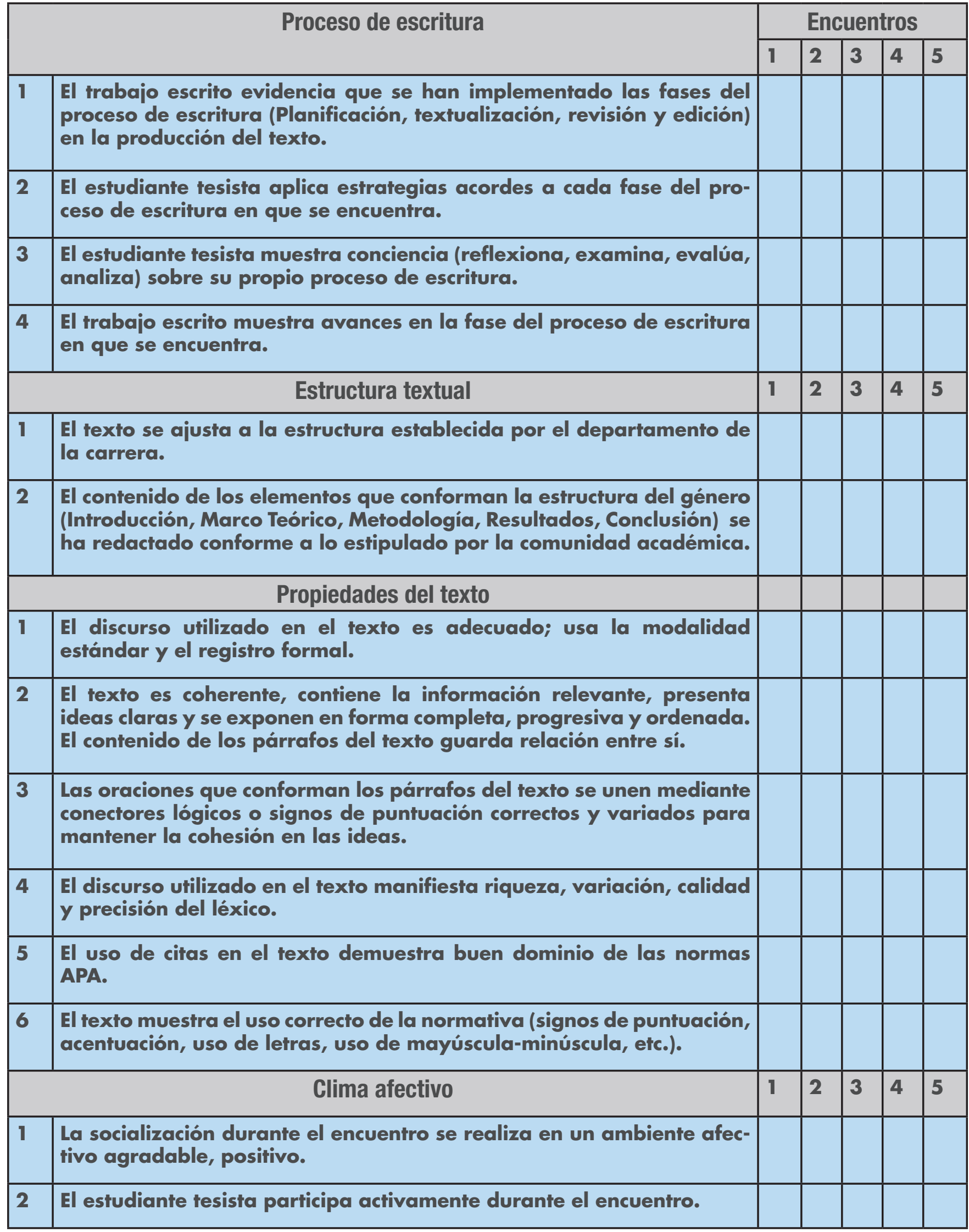


Indicadores a valorar después del acompañamiento:

\begin{tabular}{|c|c|c|c|c|c|c|}
\hline & Actitud reflexiva & 1 & 2 & 3 & 4 & 5 \\
\hline & studiante tesista & & & & & \\
\hline 1 & $\begin{array}{l}\text { Muestra apertura y actitud positiva hacia el seguimiento recibido y } \\
\text { hacia las observaciones realizadas. }\end{array}$ & & & & & \\
\hline 2 & $\begin{array}{l}\text { Muestra capacidad de reflexión y análisis durante el seguimiento a } \\
\text { su trabajo de producción escrita. }\end{array}$ & & & & & \\
\hline 3 & Acepta y retoma sugerencias. & & & & & \\
\hline 4 & $\begin{array}{l}\text { Pone en práctica las diferentes estrategias propuestas por el profesor } \\
\text { y el estudiante-tutor durante el seguimiento. }\end{array}$ & & & & & \\
\hline
\end{tabular}

Fuente: CEDILE

Además de este instrumento, utilizado por los tutores para apoyar el trabajo de grado de los estudiantes de las diferentes carreras, también se dio seguimiento a cada tutor a través de un miembro del Equipo Técnico del CEDILE, con el propósito de garantizar que los procesos se realizaran con responsabilidad y de acuerdo a los lineamientos establecidos para el programa piloto. El acompañamiento consistió en mantener una comunicación efectiva en la que, tanto el tutor como el especialista del CEDILE, velaban por la calidad de los trabajos realimentados. Con esto, se logró establecer una visión de conjunto entre todos los actores $\mathrm{y}$, al mismo tiempo, fueron creados espacios de reflexión para fortalecer las competencias profesionales de los tutores.

\section{Rutina del acompañamiento}

Los encuentros entre tutores y estudiantes-tesistas se llevaron a cabo en tres momentos (planificación, ejecución y evaluación), con una duración de una hora en total para cada encuentro, tal como se dijo anteriormente. Cada vez, se implementaron los siguientes pasos:

1. Planificación (5-10 minutos). El objetivo de este espacio consistió en identificar y clarificar los propósitos del encuentro entre tesista y tutor.

2. Ejecución (40 minutos). Dependiendo de la etapa de producción escrita en que se encontraba el tesista (planificación, textualización, revisión o edición), el tutor colaboraba, proponiendo diversas estrategias para favorecer la mejora del trabajo final de grado.

3. Evaluación (5-10 minutos). En esta tutoría, se motivaba al estudiante para que reflexionara sobre las estrategias implementadas; se establecían consensos y acciones de seguimiento para fortalecer el proceso de escritura, además de los acuerdos para el próximo encuentro.

\section{Realimentación de los trabajos escritos en formato de comentarios}

Las investigaciones realizadas que abordan el tema de la intervención de un experto durante el proceso de elaboración de una tesis establecen la complejidad de este género académico, en el que los estudiantes deben demostrar que son capaces de pensar y escribir empleando los códigos de la comunidad en la que se han formado (Tapia-Ladino, Arancibia, \& Correa, 2016).

En este sentido, la figura del tutor de este programa piloto de escritura fue creada con el propósito de ayudar a los estudiantes de las diferentes carreras para que reflexionaran sobre su escrito, es decir, para que ellos mismos pudieran darse cuenta de los problemas a enfrentar y así aprender nuevas estrategias para desarrollar su trabajo. De acuerdo a Tapia-Ladino et al., (2016), este proceso de revisión durante la escritura puede adoptar diversas formas: oral o escrita, individual o grupal, reuniones o evaluación formativa con apoyo de una rúbrica, entre otras.

Según estas autoras, uno de los recursos empleados para la revisión de la tesis es el comentario escrito (CE) que el profesor o tutor registra en los borradores de los estudiantes.

En efecto, los tutores acompañantes recibieron formación por medio de diferentes talleres denominados "acompañamiento reflexivo", en los cuales se analizaban algunos trabajos realimentados por ellos bajo el formato de comentarios escritos, con el objetivo de aportar al fortalecimiento de sus competencias como tutores. Con el propósito de revisar si proponían mejoras que se dirigían más a la forma que al contenido. Estos talleres tenían el objetivo de fortalecer sus competencias como tutores. 


\section{Acciones pedagógicas implementadas con los profesores asesores}

Para lograr que todos los procesos realizados impactaran positivamente en la calidad de los trabajos de grado de los estudiantes tesistas, se organizó una serie de talleres para los profesores ( 5 sesiones de 3 horas cada uno), siguiendo las líneas desarrolladas en el Programa de Alfabetización Académica de la PUCMM (Montenegro, 2016).

En estos encuentros se desarrollaron temas relacionados con el proceso de la escritura: definición del género, estructura y rúbrica de evaluación. Todos estos instrumentos fueron consensuados con los profesores de cada departamento: Comunicación Social, Arquitectura y Gestión Financiera y Auditoría, lo que posibilitó que el trabajo conjunto con los tutores se apoyara sobre bases sólidas. Asimismo, se mantuvo comunicación permanente entre profesores asesores, estudiantes tutores y Equipo técnico del Centro a fin de asegurar que los procesos se realizaran con calidad.

\section{Participantes del Programa piloto de escritura}

Tabla 4: Participantes del Programa piloto de escritura

\begin{tabular}{|l|l|}
\hline Cantidad de departamentos & 3 \\
\hline Cantidad de estudiantes impactados & 86 \\
\hline $\begin{array}{l}\text { Total de trabajos (algunos por equi- } \\
\text { pos de } 2 \text { estudiantes) }\end{array}$ & 55 \\
\hline Profesores asesores & 26 \\
\hline Total tutores formados & 17 \\
\hline Equipo técnico & 5 \\
\hline
\end{tabular}

Fuente: CEDILE

\section{Impacto del programa}

A continuación presentamos los puntos que hemos identificado como fortalezas del programa, los cuales apuntan directamente a los objetivos que nos propusimos. También, exponemos las debilidades que hemos identificado como oportunidades de mejora para una siguiente implementación.

\section{Fortalezas:}

1. Definición del género y su estructura por parte de los profesores asesores, en consenso con el CEDILE.

2. Uso de rúbrica para la evaluación del informe final de grado en cada carrera.

3. Formación de un equipo de profesores en Comunicación Social, Arquitectura Y Gestión Financiera y Auditoría que pueden contribuir al desarrollo de la autonomía profesional en sus respectivos departamentos.

4. Formación de un grupo de estudiantes tutores en la PUCMM.

5. Valoración positiva del acompañamiento por parte de los estudiantes tesistas.

6. Mejoramiento en la calidad de los trabajos finales de grado: ajuste a la estructura del género solicitado, producción escrita con mayor coherencia y precisión que antes del trabajo con tutores.

7. Mayor seguridad en el proceso de escritura por parte de los estudiantes tesistas al escribir su proyecto.

8. Evaluación de los procesos llevados a cabo con diferentes instrumentos, tanto por parte de los docentes, como de los estudiantes tutores y tesistas.

\section{Debilidades:}

- En las carreras de Comunicación Social y de Gestión Financiera y Auditoría se aprovechó mejor el tiempo asignado a cada estudiante (tutorías); no así en Arquitectura, debido a que las cargas horarias de los estudiantes dificultaban los encuentros.

- Los estudiantes tesistas sintieron mucha exigencia del trabajo.

- Algunos profesores no se involucraron totalmente en el proceso, al entender que con el apoyo del tutor era suficiente.

- Por asuntos de organización, algunos estudiantes no pudieron ser acompañados en la primera fase del proceso de escritura (planificación).

- A pesar de haber consensuado la estructura del informe final de grado y la rúbrica de evaluación, algunos docentes no las presentaron al inicio de la elaboración del trabajo.

- Al principio, la comunicación entre profesores asesores y tutores fue poco efectiva; luego se afinó.

- Algunos trabajos fueron orientados por los profesores con procesos anteriores a los ejecutados en el programa piloto (es decir, menos procesualmente), por lo que no mostraron la calidad esperada. 
A manera de ejemplo, se presenta la estructura de informe profesional consensuada con los profesores de Comunicación Social.

Tabla 5: Estructura del informe final de grado de Comunicación Social

\begin{tabular}{|c|c|c|c|}
\hline Claves & Aspectos & Descripción & Se relaciona con: \\
\hline $\begin{array}{l}\text { Rótulo, nombre, } \\
\text { enunciado, causa, } \\
\text { razón, pretexto. }\end{array}$ & Título & $\begin{array}{l}\text { Refleja lo que se hará. Síntesis del objetivo general. } \\
\text { Enunciado de la temática del estudio. Indica el } \\
\text { contenido. Solución al problema planteado. }\end{array}$ & $\begin{array}{l}\text {...el objetivo general. Un } \\
\text { buen título es ya un buen } \\
\text { proyecto, sobre todo, si } \\
\text { tiene subtítulo }\end{array}$ \\
\hline $\begin{array}{l}\text { Presentación, pref- } \\
\text { acio, prólogo. }\end{array}$ & Contextualización & $\begin{array}{l}\text { Presenta el documento. Hace un comentario analítico } \\
\text { del índice. Lo necesario para entrar de lleno en un } \\
\text { asunto. }\end{array}$ & ...la tabla de contenido \\
\hline \multicolumn{4}{|c|}{ ASPECTOS INTRODUCTORIOS } \\
\hline $\begin{array}{l}\text { Necesidad, vacío } \\
\text { de conocimiento, } \\
\text { datos } \\
\text { contradictorios. } \\
\text { Explicación de } \\
\text { fenómenos, } \\
\text { oportunidad, } \\
\text { requerimiento, } \\
\text { deficiencia, } \\
\text { dificultad, cuestión. }\end{array}$ & \begin{tabular}{|l|} 
Formulación del prob- \\
lema (qué)
\end{tabular} & $\begin{array}{l}\text { Indica qué respuestas deben encontrarse con el estudio. } \\
\text { Diferencia causas (variables independientes) de efectos } \\
\text { (variables dependientes). Relaciona entre dos o más } \\
\text { variables. Se caracteriza por preguntas precisas o } \\
\text { descripción de la situación problemática. Compara la } \\
\text { realidad con lo deseado. Tiene origen en la realidad } \\
\text { y conocimiento de la misma. Evidencia situación } \\
\text { deficiente o negativa. Identifica qué es, por qué sucede, } \\
\text { desde cuándo, con qué, dónde. Descompone en partes } \\
\text { e identifica variables. Se fundamenta en el análisis de } \\
\text { los ejemplos que caracterizan la situación problema. } \\
\end{array}$ & $\begin{array}{l}\text {...las situaciones } \\
\text { problemáticas identificadas. } \\
\text {...los ejemplos "malos", } \\
\text { pues ayuda a caracterizar } \\
\text { los problemas o situaciones } \\
\text { deficitarias }\end{array}$ \\
\hline $\begin{array}{l}\text { Aportes de estu- } \\
\text { dios realizados. }\end{array}$ & Antecedentes & $\begin{array}{l}\text { Revisa el estado de desarrollo del conocimiento. } \\
\text { Incluye resultados de estudios anteriormente realizados } \\
\text { relacionados con el tema. }\end{array}$ & $\begin{array}{l}\text {... el marco teórico y los } \\
\text { aspectos introductorios. }\end{array}$ \\
\hline $\begin{array}{l}\text { Acotar, precisar, } \\
\text { limitar. }\end{array}$ & $\begin{array}{l}\text { Delimitación del prob- } \\
\text { lema (hasta dónde) }\end{array}$ & $\begin{array}{l}\text { Establece límites temporales, espaciales, económicos, } \\
\text { sociales, culturales, tecnológicos, conceptuales, etc. }\end{array}$ & $\begin{array}{l}\text {....las unidades de } \\
\text { observación (personas o } \\
\text { cosas) }\end{array}$ \\
\hline $\begin{array}{l}\text { Importancia, } \\
\text { aporte, beneficio, } \\
\text { conveniencia, } \\
\text { relevancia, } \\
\text { novedad, } \\
\text { pertinencia, } \\
\text { factibilidad, } \\
\text { viabilidad, valor } \\
\text { teórico, utilidad } \\
\text { metodológica. }\end{array}$ & Justificación (por qué) & $\begin{array}{l}\text { Expone razones, beneficios que se alcanzan. Sustenta, } \\
\text { convence, motiva para la aprobación y patrocinio. } \\
\text { Demuestra por qué debe hacerse el estudio. }\end{array}$ & $\begin{array}{l}\text {...la resolución del prob- } \\
\text { lema y con las conclusiones } \\
\text { finales }\end{array}$ \\
\hline $\begin{array}{l}\text { Meta, propósito, } \\
\text { producto, logro, } \\
\text { fin. }\end{array}$ & $\begin{array}{l}\text { Objetivo } \\
\text { general } \\
\text { (para qué) } \\
\text { Avanzar, facilitar, } \\
\text { contribuir, colaborar, } \\
\text { fomentar, motivar, } \\
\text { desarrollar. }\end{array}$ & $\begin{array}{l}\text { Determina el propósito final (para qué) y meta (qué). } \\
\text { Más teórico que operativo. Puede haber uno o múltiples } \\
\text { generales. }\end{array}$ & $\begin{array}{l}\text {...la resolución del prob- } \\
\text { lema y con las conclusiones } \\
\text { finales. }\end{array}$ \\
\hline $\begin{array}{l}\text { Análisis, } \\
\text { determinación, } \\
\text { evaluación. }\end{array}$ & $\begin{array}{l}\text { Objetivos } \\
\text { específicos (para } \\
\text { qué) } \\
\text { Comparar, reconocer, } \\
\text { precisar, explicar, } \\
\text { comprobar, } \\
\text { comprender, discernir, } \\
\text { descomponer, separar. }\end{array}$ & $\begin{array}{l}\text { Señala acciones concretas, actividades para el } \\
\text { logro del objetivo general. Desglose de los objetivos } \\
\text { generales. Relación con las variables del problema. } \\
\text { Son operativos, concretos, medibles. Se reflejan en la } \\
\text { tabla de contenidos. Desarrollan de manera exhaustiva } \\
\text { el objetivo general. }\end{array}$ & $\begin{array}{l}\text {... Para cada objetivo } \\
\text { específico, diseñar su } \\
\text { metodología y su recurso. } \\
\text {...la programación de } \\
\text { actividades: cronograma }\end{array}$ \\
\hline
\end{tabular}




\section{Proceso de acompañamiento de tutores a tesistas}

\section{Evaluación cualitativa del acompañamiento realizado por estudiantes tesistas a sus tutores}

Los acompañamientos, generalmente, provocan una resistencia inicial. Al ser acompañados podemos sentir que alguien nos está vigilando, nos sentimos indefensos ante las debilidades que puedan evidenciarse. Esta sensación inicial puede superarse con la intersubjetividad que muestre el acompañante, por el apoyo genuino que ofrezca y la manera en que oriente la cognición compartida y los acuerdos para un logro común.

La respuesta al programa de acompañamiento por parte de los estudiantes tesistas fue muy positiva, ya que los tutores se distinguieron por la relación empática y positiva basada en la confianza. Los tesistas valoraron la experiencia como muy provechosa y consideraron que sus trabajos mejoraron favorablemente, tal como se puede apreciar en los siguientes comentarios:

"El plan es muy interesante y de gran ayuda para realizar el trabajo de fin de grado, ya que los estudiantes llegamos perdidos en cuanto a la manera correcta o la más adecuada a la hora de expresar las ideas vía papel. Mi tutor fue el mejor de todos; siempre estaba pendiente de las reuniones, fue muy puntual e iba preparado con material de apoyo para enriquecer nuestro escrito. También fue muy preciso con las correcciones.

Este proyecto me ha enseñado en el ámbito laboral a identificar el impacto que tienen las palabras escritas y que, si estas no se expresan de la forma correcta, se puede mal interpretar la idea central. Asimismo, he aprendido a utilizar nexos. Espero que para el período que sigue (enero-abril 2017), mi grupo sea privilegiado e incluido en el proyecto".

\section{María Pichardo, Arquitectura}

"Realmente fue una grata experiencia y el valor de las tutorías es incalculable, ya que nos ayudaron a enriquecer nuestras habilidades en cuanto a producción escrita se refiere; por consiguiente, exhorto a que el programa se expanda a otras carreras profesionales para que también puedan beneficiarse de esta excelente iniciativa".

\section{Diosmel Peña, GFA}

"No negaré que al principio no quería ser parte del programa por la escasez de tiempo en el semestre. Sabía que no iba a ser fácil, especialmente en mi caso, que ya había pasado por el proceso de redacción. Sin embargo, luego de entrar al programa, me di cuenta lo necesario que era.

Mi informe de grado cambió y la calidad que tiene ahora es mucho mejor que la de antes. En pocas palabras diré que esto me ayudó a mejorar en todos los sentidos; no solo en el proceso de la redacción de tesis, sino también en el ámbito personal. Aprendí mucho y sé que estos conocimientos me servirán en el futuro".

Yolenny Bueno, Arquitectura

Por otro lado, para determinar el impacto del trabajo realizado con los estudiantes tesistas, a los estudiantes tutores, se les entregó un instrumento con diferentes criterios sobre el proceso de acompañamiento, que se detalla a continuación:

\section{Evaluación cuantitativa del acompañamiento realizado por estudiantes tesistas a sus tutores}

Evalúa el proceso de acompañamiento que recibiste para la producción del trabajo final de grado, utilizando la siguiente escala de valoración:

\section{L=logrado, \\ $M L=$ medianamente logrado \\ $E L=$ escasamente logrado \\ $\mathrm{NL}=$ no logrado.}

Marca con un cotejo $(\sqrt{ })$ la columna que contenga la/s letra/s que representa tu valoración. Al final escribe, de ser necesario, sugerencias para mejorar dicho trabajo. 
Tabla 6: Criterios de evaluación del acompañamiento

\begin{tabular}{|c|c|c|c|c|}
\hline Criterios e indicadores a evaluar & Logrado & $\begin{array}{l}\text { Medianamente } \\
\text { logrado }\end{array}$ & $\begin{array}{l}\text { Escasamente } \\
\text { logrado }\end{array}$ & $\begin{array}{l}\text { No } \\
\text { logrado }\end{array}$ \\
\hline \multicolumn{5}{|l|}{ Antes del acompañamiento } \\
\hline $\begin{array}{l}\text { 1. El estudiante tutor se comunicó con anterioridad al comienzo del trabajo, } \\
\text { presentando el proceso que llevaría a cabo y definiendo su rol de acompañante. }\end{array}$ & $100 \%$ & & & \\
\hline 2. En el primer encuentro con el tutor, se clarificaron los propósitos del encuentro. & $100 \%$ & & & \\
\hline \multicolumn{5}{|l|}{ Durante del acompañamiento } \\
\hline $\begin{array}{l}\text { 1. El estudiante tutor apoyó el proceso de escritura del trabajo final de grado con } \\
\text { realimentación pertinente, contribuyendo así a la mejora del mismo. }\end{array}$ & $85 \%$ & $15 \%$ & & \\
\hline $\begin{array}{l}\text { 2. El apoyo brindado por el tutor te ayudó a reflexionar sobre el proceso de la } \\
\text { escritura para producir cambios significativos en la producción del trabajo final } \\
\text { de grado. }\end{array}$ & $92 \%$ & $8 \%$ & & \\
\hline 3. La revisión del trabajo se realizó durante el acompañamiento presencial. & $61 \%$ & $31 \%$ & $8 \%$ & \\
\hline \multicolumn{5}{|l|}{ Después del acompañamiento } \\
\hline 1. El tutor siempre motivó la reflexión sobre las estrategias implementadas. & $85 \%$ & $15 \%$ & & \\
\hline $\begin{array}{l}\text { 2. Se establecieron consensos para fortalecer la producción escrita del trabajo final } \\
\text { de grado. }\end{array}$ & $85 \%$ & $15 \%$ & & \\
\hline 3. Las diferentes estrategias sugeridas por el tutor fueron incorporadas al trabajo. & $85 \%$ & $15 \%$ & & \\
\hline $\begin{array}{l}\text { 4. La socialización durante el acompañamiento se realizó en un ambiente afectivo, } \\
\text { agradable, positivo. }\end{array}$ & $100 \%$ & & & \\
\hline $\begin{array}{l}\text { 5. El apoyo brindado por el tutor en el proceso de producción del trabajo de grado } \\
\text { facilitó su elaboración. }\end{array}$ & $92 \%$ & $8 \%$ & & \\
\hline
\end{tabular}

Fuente: CEDILE

A continuación, se presenta la figura 1 con la sistematización de las respuestas ofrecidas por los tesistas con los criterios mencionados anteriormente.

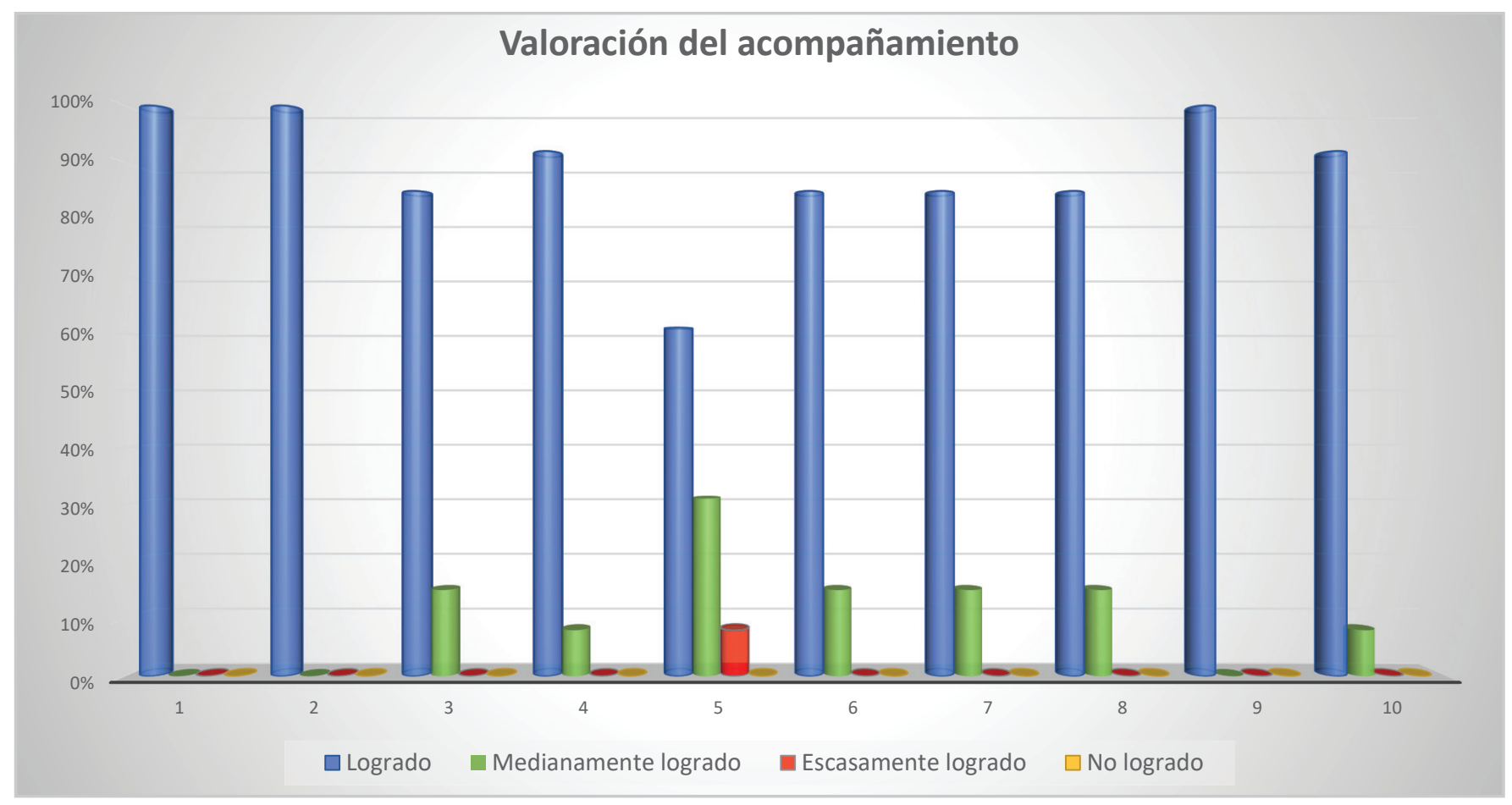

Figura 1: Instrumento de evaluación aplicado a estudiantes tesistas (Fuente: CEDILE) 
En esta figura puede notarse el impacto del programa de acompañamiento a través de las tutorías ofrecidas a los estudiantes, pues los indicadores sobre la ayuda recibida durante el proceso de elaboración del informe profesional fueron valorados, en su mayoría, entre 85 y $100 \%$.

Puede evidenciarse que los que tuvieron una mayor valoración por parte de los estudiantes fueron los relacionados a la comunicación efectiva y a la definición del rol de acompañante, o sea, a la clarificación de los propósitos; es decir, en qué consistía el acompañamiento, así como el ambiente afectivo-positivo en que este se desarrolló, todos valorados en un (100\%). De igual modo, se valoró muy alto el apoyo brindado por el tutor provocando la reflexión para que el mismo tesista fuera consciente de la mejora que debía producir en su trabajo (92\%).

Aunque un $8 \%$ consideró que la revisión del trabajo no se realizó presencialmente, pudo deberse a que, en ocasiones, por las diferentes cargas horarias de los tesistas, estos no disponían del tiempo necesario para reunirse con sus tutores, por lo que el acompañamiento se realizaba de forma virtual.

Otro criterio que se les solicitó evaluar a los estudiantes tesistas fue la asesoría que recibieron del profesor disciplinar que, como ya se dijo anteriormente, participaron en un proceso formativo ofrecido por el Centro y cuyos criterios se detallan a continuación:

\section{Evaluación cuantitativa del rol desempeñado por el profesor por parte de los estudiantes tesistas}

Tabla 7: Evaluación del desempeño del profesor

\begin{tabular}{|c|c|c|c|c|}
\hline \multicolumn{5}{|l|}{ Asesoría del profesor } \\
\hline Criterios a evaluar & Logrado & \begin{tabular}{|l|}
$\begin{array}{l}\text { Medianamente } \\
\text { logrado }\end{array}$ \\
\end{tabular} & \begin{tabular}{|l|} 
Escasamentie \\
logrado
\end{tabular} & \begin{tabular}{|l|} 
No \\
logrado
\end{tabular} \\
\hline $\begin{array}{l}\text { 1. El apoyo brindado por el profesor asesor contribuyó a } \\
\text { mejorar el proceso de elaboración del trabajo final de grado. }\end{array}$ & $92 \%$ & $8 \%$ & & \\
\hline $\begin{array}{l}\text { 2. El profesor asesor explicó el programa piloto y el apoyo que } \\
\text { recibirías de un tutor/a. }\end{array}$ & $69 \%$ & $31 \%$ & & \\
\hline $\begin{array}{l}\text { 3. El profesor asesor modeló las estrategias del proceso de } \\
\text { escritura: planificación, textualización, revisión y edición. }\end{array}$ & $46 \%$ & $31 \%$ & $23 \%$ & \\
\hline $\begin{array}{l}\text { 4. La estructura de trabajo final de grado fue presentada por } \\
\text { el profesor asesor con anterioridad al proceso de escribir y } \\
\text { contenía los mismos indicadores que la estructura presentada } \\
\text { por el tutor. }\end{array}$ & $85 \%$ & $15 \%$ & & \\
\hline $\begin{array}{l}\text { 5. Durante el proceso de escritura, el profesor asesor ofreció } \\
\text { diferentes estrategias para la producción y revisión del } \\
\text { trabajo final de grado. }\end{array}$ & $62 \%$ & $23 \%$ & $15 \%$ & \\
\hline $\begin{array}{l}\text { 6. El profesor asesor presentó la rúbrica de evaluación del } \\
\text { trabajo final de grado antes de iniciarse el proceso de } \\
\text { escritura. }\end{array}$ & $77 \%$ & $8 \%$ & $15 \%$ & \\
\hline
\end{tabular}

Fuente: CEDILE 
En la siguiente figura puede observarse la sistematización de los resultados de las valoraciones estudiantiles a la asesoría del profesor en cuanto al trabajo escrito.

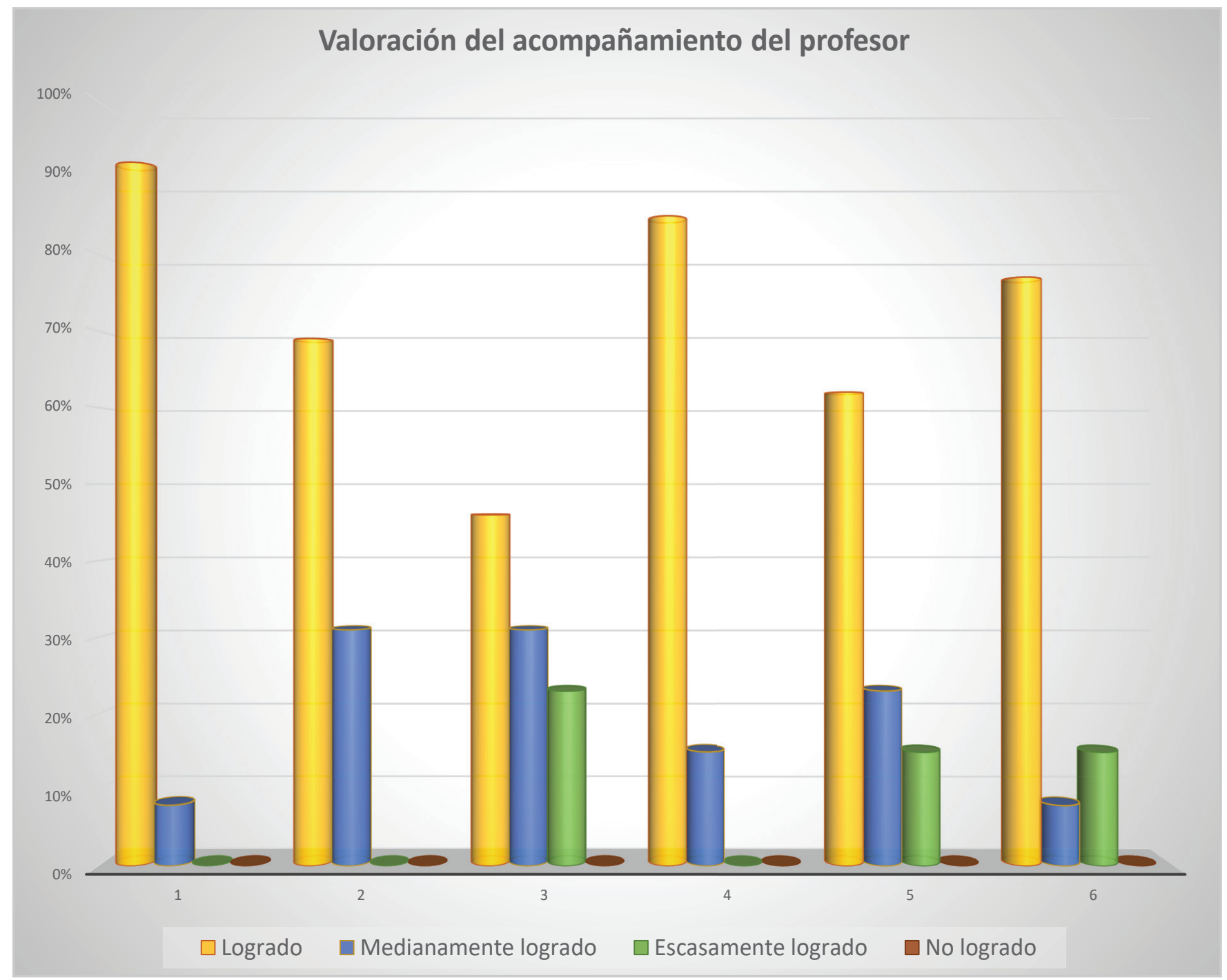

Figura 2: Instrumento de evaluación a la asesoría del profesor en el trabajo escrito (Fuente: CEDILE)

En este gráfico puede notarse que los tres indicadores mejor valorados (de 75 a 92\%) fueron el 1, el 4 y el 6 . En cuanto a los indicadores 2, 3 y 5 , que no fueron bien valorados (42 a 68\%), se observa que el 3 y el 5 se relacionaron con la transposición didáctica del docente respecto al proceso de escritura. Se esperaba que las estrategias aprendidas en los talleres realizados fueran modeladas por los profesores asesores.

\section{Conclusiones:}

El programa piloto llevado a cabo en la PUCMM para apoyar la escritura de los trabajos finales de grado en Comunicación Social, Arquitectura Y Gestión Financiera y Auditoría ha revelado ser una experiencia innovadora y beneficiosa, tanto para los docentes asesores, como para los estudiantes tesistas. En efecto, la colaboración interdisciplinar (CEDILE y cada uno de los departamentos involucrados) contribuyó al dominio de un género del discurso, cuya finalidad es comunicar conocimientos.

Por esta razón, quien lo escribe (en este caso el estudiante tesista), necesita recibir el apoyo de un experto para utilizar las estrategias de escritura apropiadas a su producción. Cabe señalar que es a partir de esta publicación que los estudiantes pasan a formar parte de la comunidad profesional de su disciplina, pudiendo ser su trabajo final un referente para otras investigaciones. 
Es de vital importancia la implementación de este tipo de programa para el nivel superior, debido a que se estaría contribuyendo a la producción científica en los diferentes campos.

De esta manera, se conforman grupos en el contexto universitario que generan diferentes formas de producir discursos para luego ponerlos en circulación. Pero para lograr resultados favorables se necesita de un equipo bien formado que tenga claridad sobre el rol que desempeña y así estimular las competencias necesarias a partir de la reflexión sobre lo que se escribe, lo que contribuirá con la formación de nuevos escritores que se convertirán en participantes activos de la comunidad discursiva de su disciplina.

Por otro lado, Es evidente el cambio de paradigma que necesitamos en cuanto a la enseñanza de la producción escrita disciplinar, lo cual es un reto para la comunidad académica, ya que, muchas veces se cuenta con la teoría; sin embargo, realizar la transformación didáctica en el aula implica innovación y hacerse consciente de la importancia de ofrecer a los estudiantes las herramientas necesarias para convertirse en escritores autónomos.

En ese sentido, es necesario continuar las propuestas de formación a los docentes y, al mismo tiempo, acompañarles en el proceso de apropiación de nuevas estrategias para favorecer la competencia de escritura en los estudiantes.

Al concluir el programa piloto se espera analizar detalladamente los trabajos finales y así determinar con precisión cuántos informes, de los que fueron favorecidos con las tutorías, mejoraron considerablemente la calidad en su producción escrita. Asimismo, continuar el afinamiento de todos los procesos desarrollados para seguir cooperando con la comunidad universitaria.

\section{Referencias}

Carlino, P. Iglesia, I. Laxalt, I. (2013). Concepciones y prácticas declaradas de profesores terciarios en torno al leer $y$ escribir en las asignaturas. Revista de Docencia Universitaria. Vol.11 (1) 105135. Buenos Aires, Argentina. Recuperado de : https://polipapers.upv.es/index.php/REDU/article/ view/5594

Carlino, P. (2011). La escritura en la investigación. Recomendaciones para elaborar una tesis. Guía para estudiantes de posgrado. Universidad de Colima, México. Editorial, PRED
Cassany, D. (2003). Describir el escribir. Cómo se aprende a escribir. Barcelona, Paidós

Giudice, J., Godoy, M., y Moyano, E. (2016). Prácticas de lectura y escritura en el marco de la enseñanza de la Psicología: profesores-investigadores en la Universidad de Flores, Facultad de Psicología y Ciencias Sociales. Pedernera 275, Ciudad Autónoma de Buenos Aires. http://www.redalyc. org/articulo.oa?id=14045395007

Molina, V. (2016). Resolución de conflictos en las tutorías de escritura según la teoría pragmadialéctica. http:// www.scielo.org.co/pdf/folios/n45/n45a02.pdf

Montenegro, L. (2016). Leeryescribiren el nivel superior: Lecciones aprendidas y desafíos del Programa Institucional de Alfabetización Académica llevado a cabo con tres cohortes de profesores. Obtenido de http://writingprogramsworldwide.ucdavis.edu/: http://writing programsworldwide.ucdavis.edu/ index.php/dominican-republic/

Moyano, E., Natale, L. (2012). La enseñanza de la lectura y la escritura como política institucional a lo largo de las carreras universitarias. El caso de la Universidad Nacional de General Sarmiento. Argentina.

Tapia-Ladino, Arancibia, B., y Correa, R. (2016). Rol de los comentarios escritos en la construcción de la tesis desde la perspectiva de estudiantes tesistas y profesores guía. Universitas Psychologica, Colombia. 